\title{
Estudo do metabolismo da glicose na tuberculose pulmonar ativa utilizando a tomografia por emissão de pósitrons (18F-FDG PET)*
}

\author{
Sidney BOMBARdA ${ }^{1}$, JOSÉ SOARES JÚNIOR², MÁRIO TERRA FILHO ${ }^{3}$
}

\begin{abstract}
Os métodos de imagem utilizados na avaliação da tuberculose pulmonar incluem a radiografia e a tomografia computadorizada do tórax. As imagens obtidas pelos métodos de medicina nuclear permitem estudos funcionais e metabólicos dos órgãos de interesse, através do uso de radiofármacos específicos. Alterações do metabolismo da glicose podem ser detectadas pela tomografia por emissão de pósitrons (PET) utilizando-se o 18F-fluorodesoxiglicose (18F-FDG). Essas alterações estão presentes

nas doenças neoplásicas, inflamatórias e infecciosas. A tuberculose é uma doença granulomatosa causada pelo Mycobacterium tuberculosis, que se utiliza de glicose como fonte de energia. Objetivo: O estudo do metabolismo da glicose na tuberculose pulmonar através da PET e sua comparação com a tomografia computadorizada de tórax. Material e métodos: Foram avaliados 20 pacientes portadores de tuberculose pulmonar. Todos foram submetidos à PET e à tomografia computadorizada de tórax, em até 30 dias após o início do tratamento. Resultados: Todos os pacientes apresentaram captação positiva do 18F-FDG na PET. Na tomografia computadorizada do tórax, todos os pacientes apresentaram sinais compatíveis com atividade de tuberculose. A sensibilidade dos dois métodos foi de $100 \%$. Houve concordância entre os achados do 18F-FDG PET e da tomografia computadorizada ( $\mathrm{K}=$ 0,27 e p < 0,001). Conclusões: Concluiu-se, através do 18F-FDG PET, que o metabolismo da glicose está alterado na tuberculose pulmonar e que há concordância com as alterações anatômicas observadas pela tomografia computadorizada do tórax. (J Pneumol 2002;28(5):270-6)
\end{abstract}

\section{Evaluation of glucose metabolism in active lung tuberculosis by positron-emission tomography (18F-FDG PET)}

Current methods to evaluate lung tuberculosis include chest radiography and computed tomography. Nuclear medicine imaging techniques are performed after administration of specific radiopharmaceuticals that accumulate in the organs of interest. Alterations of glucose metabolism can be observed by positron-emission tomography, using 18F-fluorodeoxyglucose (18FFDG PET). These findings are present in the neoplasms, but also in inflammatory and infectious diseases. Tuberculosis is a granulomatous disease caused by Mycobacterium tuberculosis, that uses glucose as an energy source. Purpose: The study of glucose metabolism in lung tuberculosis by

PET and the comparison of this results to CT scan findings. Material and methods: Twenty patients with active lung tuberculosis were evaluated. The 18F-FDG PET and computed tomography were performed in all patients, after the diagnosis and until 30 days of treatment. Results of both methods were compared. Results: All patients showed 18F-FDG positive uptake. On the computed

tomography, all patients showed signs compatible with tuberculosis. The sensitivity of both methods was of $100 \%$. There was significant concordance between the two methods $(K=0.27)$.

Conclusions: The study concludes that glucose metabolism is modified in lung tuberculosis. There was concordance in the anatomic changes observed on computed tomography.

\footnotetext{
* Trabalho realizado na Faculdade de Medicina da Universidade de São Paulo, São Paulo, SP.

1. Assistente Doutor da Disciplina de Pneumologia.

2. Médico Chefe do Serviço de Radioisótopos do InCor.

3. Professor associado da Disciplina de Pneumologia, InCor.
}

Endereço para correspondência - Sidney Bombarda, Rua Ezequiel Freire, 35, sala 33 - 02034-000 - São Paulo, SP. Tel./fax (11) 69775227; e-mail: sbombarda@ig.com.br

Recebido para publicação em 27/3/02. Aprovado, após revisão, em 30/7/02. 
Descritores - Tuberculose. Glicose. Fluorodesoxiglucose. Tomografia computadorizada de emissão. Tomografia computadorizada. Key words - Tuberculosis. Glucose. Fluorodeoxyglucose. Emissioncomputed tomography. Computed tomography.

\section{INTRODUÇÃO}

A tuberculose é a doença infecciosa mais comum da espécie humana, acometendo 8,4 milhões de pessoas em todo o mundo em 1999. Esta incidência supera a de 1997, quando foram notificados 8 milhões de casos novos. Estima-se que, em 2005, esse número chegue a 10,2 milhões, a maioria vivendo em países emergentes ${ }^{(1)}$.

O diagnóstico da tuberculose pulmonar, em nosso meio, baseia-se no encontro de duas baciloscopias diretas positivas no escarro ou uma cultura positiva para Mycobacterium tuberculosis. Na ausência desses achados, imagens radiológicas sugestivas ou outros exames complementares associados a achados clínicos são indicativos de doença ativa ${ }^{(2)}$. $\mathrm{O}$ adequado conhecimento das imagens compatíveis com atividade da tuberculose é, portanto, um importante recurso para o seu diagnóstico.

A radiografia do tórax é o método de imagem de escolha na avaliação inicial e no acompanhamento da tuberculose pulmonar. A tomografia computadorizada do tórax (TC) é utilizada na suspeita clínica de tuberculose pulmonar, especialmente nos casos em que a radiografia inicial é normal, na diferenciação com outras doenças torácicas e em pacientes com AIDS ou com febre de origem desconhecida ${ }^{(3)}$.

As imagens obtidas pela radiografia e pela TC são representativas das alterações estruturais ou anatômicas dos órgãos ou tecidos comprometidos. No entanto, alterações bioquímicas podem estar presentes e preceder as alterações visualizadas por esses recursos diagnósticos.

Certos métodos utilizados em medicina nuclear fornecem imagens funcionais que podem ser mais sensíveis que aquelas puramente anatômicas ou estruturais. Essas imagens são obtidas utilizando-se sistemas de detecção sensíveis às radiações gama emitidas pelos radiofármacos, que se concentram nos órgãos ou tecidos de interesse.

A PET produz imagens tridimensionais e tomográficas da distribuição no organismo de radioisótopos emissores de pósitrons. O diferencial da PET, em relação aos outros métodos utilizados em medicina nuclear, é a possibilidade de marcar substâncias que fazem parte dos compostos orgânicos, permitindo, assim, estudos metabólicos do organismo.

As aplicações iniciais da PET foram direcionadas às doenças neurológicas e cardiológicas. Atualmente, a PET é indicada principalmente em oncologia, resultado do suces-
Siglas e abreviaturas utilizadas neste trabalho

TC - Tomografia computadorizada do tórax

AIDS - Síndrome da imunodeficiência humana adquirida

PET - Tomografia por emissão de pósitrons

FDG - (2[18F]-fluor-2-desoxi-D-glicose)

$\mathrm{MBq}$ - Megabecquerel

$14 \mathrm{C}$ - Isótopo 14 do carbono

so da aplicação do FDG (2[18F]-fluor-2-desoxi-D-glicose) no diagnóstico, estadiamento e seguimento de vários tipos de câncer. O FDG é um análogo da glicose marcado com o flúor-18, radioisótopo emissor de pósitrons, que permite o estudo do metabolismo da glicose, através de sua maior captação em tecidos com maior atividade glicolítica $^{(4-9)}$

Um amplo espectro de alterações bioquímicas está presente nas células tumorais, incluindo maiores taxas de glicólise aeróbia e anaeróbia quando comparadas com as encontradas em tecidos normais. Com base nessas diferenças bioquímicas, a PET utilizando o FDG é bastante sensível na detecção de câncer de pulmão, mesmo em pacientes que apresentam alterações indeterminadas na TC. A especificidade é menor que a sensibilidade devido à captação aumentada do FDG em certas doenças inflamatórias e infecciosas ${ }^{(10,11)}$.

$\mathrm{O}$ câncer de pulmão acomete preferencialmente os campos superiores pulmonares ${ }^{(12)}$. Na tuberculose do adulto, as cavitações são mais freqüentes nos segmentos ápico-posteriores dos lobos superiores ou superiores dos lobos inferiores ${ }^{(13)}$. Por esse motivo, é importante, principalmente em países onde a prevalência dessa doença granulomatosa é alta, conhecer o comportamento do 18F-FDG-PET em pacientes adultos portadores de tuberculose pulmonar, na tentativa de contribuir para o possível diagnóstico diferencial com as neoplasias de pulmão.

O objetivo deste estudo foi o de avaliar o metabolismo da glicose utilizando o 18F-FDG através da PET em pacientes portadores de tuberculose pulmonar ativa e comparar os resultados com a tomografia computadorizada do tórax (TC) realizada no mesmo momento.

\section{MATERIAL E MÉTODOS}

Foram estudados, prospectivamente, 20 pacientes portadores de tuberculose pulmonar, não diabéticos, com sorologia negativa para o vírus HIV. A tuberculose pulmonar foi confirmada por presença de Mycobacterium tuberculosis em cultura de escarro (19 pacientes) ou presença de granuloma com necrose caseosa central e cultura positiva para Mycobacterium tuberculosis em fragmento de pulmão por biópsia transbrônquica (um paciente). 
Todos os pacientes foram esclarecidos quanto aos procedimentos a serem realizados. Os pacientes foram submetidos à TC convencional realizada no Serviço de Radiologia do Instituto do Coração do Hospital das Clínicas da Faculdade de Medicina da Universidade de São Paulo e à 18F-FDG-PET realizada no Departamento de Radioisótopos do Instituto do Coração do Hospital das Clínicas da Faculdade de Medicina da Universidade de São Paulo.

A avaliação foi feita quando do diagnóstico da tuberculose e até 30 dias após o início do tratamento com esquema 1 (rifampicina, isoniazida e pirazinamida).

\section{PET}

Os pacientes foram submetidos a jejum de 12 horas. Após este período, foi colhida amostra de sangue para a determinação sérica da glicose. O FDG foi administrado na dose de 70 a $100 \mathrm{MBq}$, por via endovenosa, aproximadamente 45 minutos antes da obtenção das imagens. Durante esse período, todos os pacientes permaneceram em repouso.

As imagens foram obtidas em câmara de cintilação $A D A C$ Vertex Plus (ADAC Laboratories), tendo-se 64 quadros a cada seis graus, numa órbita de 360 graus, com duração de 40 segundos. As imagens foram reconstruídas utilizando-se o método interativo e apresentadas nos eixos sagital, coronal e transverso.

Os pulmões, para efeito de análise das imagens obtidas, foram divididos em três campos (superior, médio $e$ inferior) e cada campo foi subdividido em duas porções (anterior e posterior).

Dois observadores avaliaram as imagens obtidas.

A captação foi classificada conforme a sua intensidade em três graus:

Grau 0 ou negativa - captação de igual intensidade ou menor que a observada na região axilar anterior.

Grau 1 - a menor captação possível que pôde ser considerada maior que a observada na região axilar anterior.

Grau 2 - captação de intensidade muito maior que a observada na região axilar anterior.

\section{TC}

As TC foram realizadas em um tomógrafo convencional da marca Philips, modelo Tomoscan LX. Foi realiza$\mathrm{da}$, inicialmente, uma radiografia digital incluindo a região cervical até os hipocôndrios, para planejamento dos cortes. Foram então obtidos cortes axiais com $10 \mathrm{~mm}$ de espessura e incremento de $10 \mathrm{~mm}$ da região apical até a base dos pulmões.

Foram considerados sinais compatíveis com atividade de tuberculose: nódulos centrolobulares de distribuição segmentar, consolidações, cavidades de paredes espessas, nódulos, massas, espessamento de parede brônqui- ca, aspecto de "árvore em florescência" e bronquiecta$\operatorname{sias}^{(14-18)}$.

Os pulmões, para efeito de análise das imagens obtidas, foram divididos em três campos (superior, médio e inferior) e cada campo foi subdividido em duas porções (anterior e posterior).

Dois observadores avaliaram as imagens obtidas.

De acordo com a extensão, considerando um ou mais dos sinais compatíveis com atividade de tuberculose no campo analisado, os achados tomográficos foram classificados em três graus:

Grau 0 - ausência de sinais compatíveis com atividade de tuberculose.

Grau 1 - presença de sinais compatíveis com atividade de tuberculose em até 50\% do campo analisado.

Grau 2 - presença de sinais compatíveis com atividade de tuberculose em mais do que $50 \%$ do campo analisado.

\section{Análise estatística}

Calculou-se a sensibilidade e a especificidade de cada um dos métodos. Compararam-se as especificidades dos dois métodos através do teste de diferença de duas proporções $(p)^{(18)}$. Verificou-se a concordância entre os dois métodos através da estatística Kappa ${ }^{(18)}$.

Adotou-se o nível de significância de 5\%.

\section{Resultados}

A idade média dos pacientes foi de 34,3 anos (16 a 71 anos), com desvio padrão de 13,8 anos. Dos 20 indivíduos estudados, 11 eram do sexo masculino (55\%) e nove do feminino (45\%). Todos os pacientes apresentavam quadro clínico compatível com tuberculose. As características clínicas dos pacientes estão descritas na Tabela 1.

Todos os pacientes $(20 / 20)$ apresentaram captação positiva do FDG de grau 1 ou 2 no início do tratamento (Figuras 1, 2 e 3). À TC, todos os pacientes (20/20) apresentavam sinais compatíveis com atividade de tuberculose, de grau 1 ou 2, no início do tratamento. A sensibilidade do PET e da TC foi de 100\%.

A concordância bruta entre a PET e a TC no início do tratamento foi de $85 \%$ nos campos analisados (Tabela 2). A concordância líquida, retirando-se o efeito do acaso, foi de $37 \%$ ( $\mathrm{K}=0,3692=0,37)$. Essa concordância foi estatisticamente significativa $(p<0,001)$.

\section{DISCUSSÃO}

Em 1930, Lawrence desenvolveu o acelerador nuclear ou cíclotron e, em 1938, o mesmo Lawrence e Livingston designaram os primeiros cíclotrons chamados "médicos". A partir de então, vários radioisótopos artificiais foram produzidos e utilizados na marcação de compostos para estudos biológicos, bioquímicos e médicos ${ }^{(19)}$. 
TABELA

Características clínicas

\begin{tabular}{|c|c|c|c|c|c|c|c|c|c|c|}
\hline & Sexo & Idade & Tabagismo & Tosse & Febre & $\begin{array}{c}\text { Perda } \\
\text { de peso }\end{array}$ & $\begin{array}{c}\text { Sudorese } \\
\text { noturna }\end{array}$ & Anorexia & $\begin{array}{l}\text { Peso } \\
\text { inicial } \\
(\mathrm{kg})\end{array}$ & $\begin{array}{c}\text { Peso } \\
\text { pós-tratamento } \\
(\mathrm{kg})\end{array}$ \\
\hline 1 & M & 40 & + & + & + & + & + & + & 62,0 & 65,9 \\
\hline 2 & M & 42 & + & + & + & + & + & + & 60,2 & 64,8 \\
\hline 3 & $M$ & 39 & + & + & + & + & + & + & 53,5 & 56,5 \\
\hline 4 & $\mathrm{~F}$ & 40 & + & + & + & + & + & + & 50,3 & 54,6 \\
\hline 5 & $M$ & 44 & + & + & + & + & + & + & 62,6 & 65,2 \\
\hline 6 & $\mathrm{~F}$ & 18 & - & + & + & + & + & + & 52,8 & 54,5 \\
\hline 7 & M & 39 & - & + & + & + & + & + & 60,6 & 64,8 \\
\hline 8 & $\mathrm{~F}$ & 24 & - & + & + & + & + & + & 48,3 & 52,7 \\
\hline 9 & $\mathrm{~F}$ & 29 & - & + & + & + & + & + & 48,2 & 53,4 \\
\hline 10 & $\mathrm{~F}$ & 47 & - & + & + & + & + & + & 52,5 & 56,1 \\
\hline 11 & $\mathrm{~F}$ & 27 & - & + & + & + & + & + & 54,4 & 58,2 \\
\hline 12 & $M$ & 34 & - & + & + & + & + & + & 57,7 & 58,5 \\
\hline 13 & $M$ & 71 & - & + & + & + & + & + & 52,6 & 54,6 \\
\hline 14 & $\mathrm{~F}$ & 20 & - & + & + & + & + & + & 50,3 & 54,3 \\
\hline 15 & $\mathrm{~F}$ & 18 & - & + & + & + & + & + & 49,3 & 55,5 \\
\hline 16 & $M$ & 54 & - & + & + & + & + & - & 58,3 & 62,2 \\
\hline 17 & $M$ & 24 & - & + & + & + & + & + & 57,2 & 59,5 \\
\hline 18 & $\mathrm{~F}$ & 16 & + & + & + & + & + & + & 48,5 & 54,8 \\
\hline 19 & $M$ & 23 & + & + & + & + & + & + & 64,8 & 68,3 \\
\hline 20 & $M$ & 38 & + & + & + & + & + & + & 62,5 & 67,2 \\
\hline
\end{tabular}

TABELA 2

Resultados PET/TC

\begin{tabular}{|c|c|c|c|c|c|c|c|c|c|c|c|c|}
\hline & DSA & DMA & DIA & DSP & DMP & DIP & ESA & EMA & EIA & ESP & EMP & EIP \\
\hline 1 & $2 / 1$ & $1 / 1$ & $0 / 1$ & $1 / 1$ & $1 / 1$ & $0 / 1$ & $2 / 2$ & $2 / 1$ & $1 / 1$ & $1 / 2$ & $2 / 2$ & $2 / 1$ \\
\hline 2 & $2 / 1$ & $0 / 1$ & $0 / 0$ & $2 / 2$ & $2 / 1$ & $1 / 0$ & $0 / 1$ & $0 / 1$ & $0 / 0$ & $2 / 2$ & $2 / 2$ & $2 / 0$ \\
\hline 3 & $2 / 1$ & $2 / 1$ & $2 / 1$ & $2 / 2$ & $0 / 1$ & $0 / 0$ & $2 / 1$ & $2 / 1$ & $0 / 0$ & $0 / 0$ & $0 / 1$ & $2 / 1$ \\
\hline 4 & $2 / 1$ & $1 / 1$ & $0 / 0$ & $2 / 2$ & $1 / 1$ & $1 / 1$ & $0 / 0$ & $0 / 0$ & $0 / 0$ & $0 / 0$ & $0 / 0$ & $0 / 0$ \\
\hline 5 & $0 / 2$ & $2 / 2$ & $0 / 0$ & $1 / 0$ & $0 / 0$ & $0 / 0$ & $0 / 0$ & $0 / 0$ & $0 / 0$ & $0 / 0$ & $0 / 0$ & $0 / 0$ \\
\hline 6 & $0 / 0$ & $0 / 0$ & $1 / 2$ & $0 / 0$ & $0 / 2$ & $2 / 1$ & $1 / 1$ & $1 / 1$ & $0 / 0$ & $0 / 0$ & $0 / 0$ & $0 / 0$ \\
\hline 7 & $0 / 0$ & $0 / 0$ & $0 / 0$ & $0 / 0$ & $0 / 0$ & $0 / 0$ & $2 / 2$ & $2 / 1$ & $0 / 0$ & $0 / 0$ & $0 / 1$ & $0 / 0$ \\
\hline 8 & $0 / 0$ & $0 / 0$ & $2 / 1$ & $0 / 0$ & $1 / 1$ & $1 / 2$ & $0 / 0$ & $0 / 0$ & $0 / 0$ & $0 / 0$ & $0 / 0$ & $0 / 0$ \\
\hline 9 & $2 / 2$ & $1 / 1$ & $1 / 1$ & $1 / 2$ & $0 / 1$ & $0 / 0$ & $0 / 1$ & $2 / 1$ & $0 / 0$ & $0 / 1$ & $0 / 1$ & $0 / 0$ \\
\hline 10 & $1 / 1$ & $0 / 1$ & $0 / 0$ & $2 / 2$ & $0 / 0$ & $0 / 0$ & $0 / 1$ & $0 / 0$ & $0 / 0$ & $1 / 1$ & $0 / 1$ & $0 / 0$ \\
\hline 11 & $0 / 0$ & $1 / 1$ & $0 / 0$ & $0 / 0$ & $0 / 1$ & $0 / 0$ & $2 / 1$ & $2 / 1$ & $0 / 1$ & $0 / 2$ & $0 / 1$ & $0 / 0$ \\
\hline 12 & $0 / 0$ & $0 / 0$ & $0 / 0$ & $0 / 1$ & $0 / 1$ & $0 / 0$ & $0 / 1$ & $0 / 0$ & $0 / 0$ & $1 / 1$ & $0 / 1$ & $0 / 0$ \\
\hline 13 & $1 / 2$ & $1 / 1$ & $0 / 0$ & $2 / 2$ & $0 / 1$ & $0 / 1$ & $0 / 1$ & $1 / 1$ & $0 / 0$ & $0 / 1$ & $1 / 1$ & $0 / 1$ \\
\hline 14 & $0 / 0$ & $0 / 0$ & $0 / 0$ & $0 / 0$ & $0 / 0$ & $0 / 0$ & $0 / 0$ & $0 / 0$ & $0 / 1$ & $1 / 0$ & $0 / 2$ & $0 / 0$ \\
\hline 15 & $1 / 1$ & $0 / 1$ & $0 / 0$ & $0 / 0$ & $0 / 0$ & $0 / 0$ & $2 / 1$ & $2 / 1$ & $1 / 1$ & $2 / 2$ & $2 / 1$ & $0 / 0$ \\
\hline 16 & $1 / 1$ & $1 / 0$ & $1 / 0$ & $1 / 1$ & $1 / 0$ & $1 / 0$ & $0 / 1$ & $0 / 0$ & $0 / 0$ & $2 / 2$ & $0 / 1$ & $0 / 0$ \\
\hline 17 & $0 / 0$ & $0 / 0$ & $0 / 0$ & $2 / 2$ & $1 / 2$ & $0 / 0$ & $0 / 0$ & $0 / 0$ & $0 / 0$ & $0 / 0$ & 0/0 & $0 / 0$ \\
\hline 18 & $0 / 0$ & $1 / 0$ & $1 / 1$ & $2 / 0$ & $0 / 2$ & $0 / 1$ & $0 / 0$ & $1 / 1$ & $0 / 1$ & $0 / 0$ & $0 / 0$ & $0 / 0$ \\
\hline 19 & $1 / 2$ & $0 / 0$ & $0 / 0$ & $1 / 2$ & $0 / 1$ & $0 / 0$ & $0 / 0$ & $0 / 0$ & $0 / 0$ & $0 / 0$ & $0 / 0$ & $0 / 0$ \\
\hline 20 & $1 / 1$ & $0 / 1$ & $0 / 1$ & $0 / 2$ & $1 / 2$ & $0 / 0$ & $2 / 1$ & $0 / 1$ & $0 / 0$ & $0 / 1$ & $0 / 1$ & $0 / 0$ \\
\hline
\end{tabular}

Legenda: $\mathrm{D}=$ Direito; $\mathrm{E}=$ Esquerdo; $\mathrm{S}=$ Superior; $\mathrm{M}=$ Médio; $\mathrm{I}=$ Inferior $\mathrm{A}=$ Anterior $\mathrm{P}=$ Posterior

$\square$ Campos concordantes

Campos discordantes 


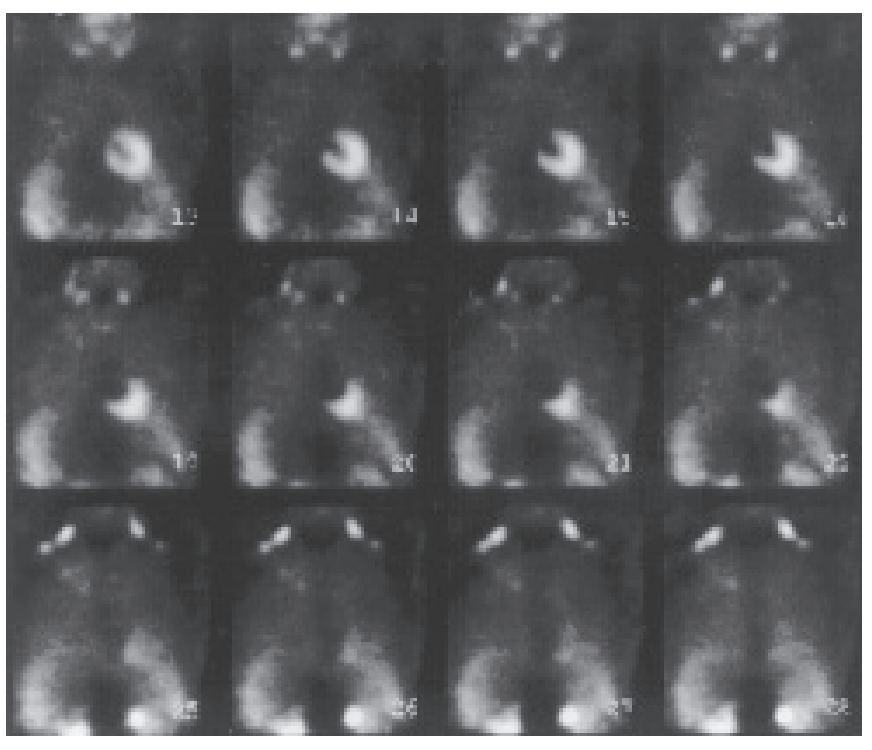

Figura 1 - Captação de grau 0: as imagens do corte coronal do PET evidenciam captação do 18 F-FDG nos pulmões de igual intensidade à observada nas regiões axilares anteriores (normal). Observa-se captação aumentada do radiofármaco em coração, rins e músculos da região cervical (fisiológica).

A PET é uma técnica de imagem que se utiliza de moléculas radiomarcadas para o estudo de suas interações nos processos biológicos, in vivo.

Em 1977, Sokoloff et al. descreveram o clássico papel do 14C-desoxiglicose na avaliação da utilização da glicose no cérebro(19). O fato de o $14 \mathrm{C}$ apresentar meia-vida muito curta limitava o seu uso. A utilização, a partir de 1995, da desoxiglicose marcada com $18 \mathrm{~F}$, elemento de meiavida de 110 minutos, possibilitou a adoção da PET como recurso diagnóstico nas doenças que cursam com alterações no metabolismo da glicose, especialmente as doenças neoplásicas ${ }^{(20)}$.

Assim, o FDG tem sido útil no diagnóstico e seguimento de vários tipos de câncer. Entretanto, outras doenças, tanto inflamatórias quanto infecciosas, apresentam aumento da captação do FDG, dificultando a diferenciação entre doença benigna e maligna.

Alguns estudos in vitro demonstram acúmulo do FDG em leucócitos, linfócitos e macrófagos ${ }^{(8,21)}$. Dessa forma, processos inflamatórios mediados por essas células podem apresentar captação aumentada do FDG através da PET.

Nas doenças infecciosas, o acúmulo do FDG ocorre devido à presença de células inflamatórias migratórias, do tecido de granulação ou do microorganismo causador ${ }^{(8)}$. No presente estudo, todos os pacientes apresentaram captação aumentada do FDG nos pulmões acometidos pela tuberculose.

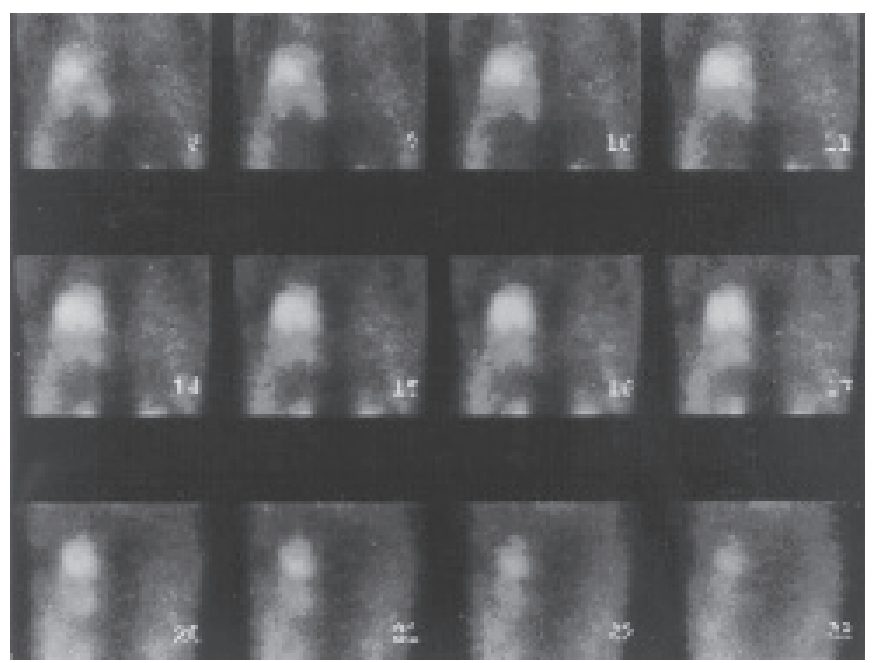

Figura 2 - Captação de grau 1: observa-se, nos cortes coronais do PET, captação do 18F-FDG no pulmão direito de intensidade maior que a vista nas regiões axilares anteriores.

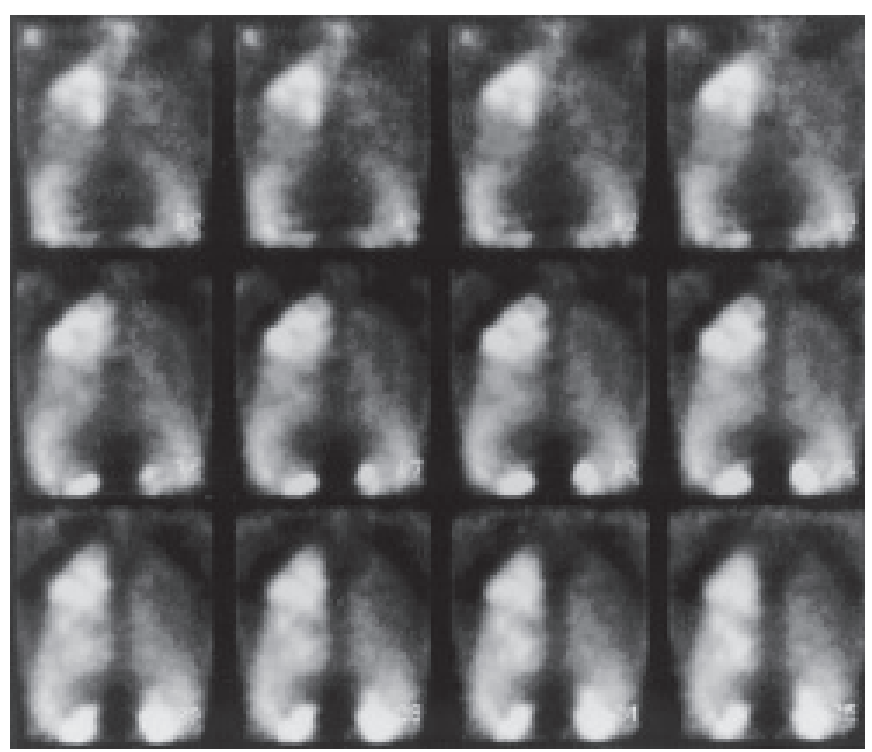

Figura 3 - Captação de grau 2: nas imagens do PET (cortes coronais), observa-se hipercaptação do 18F-FDG nas porções superior e média do pulmão direito, de intensidade muito maior que a vista nas regiões axilares anteriores. Captação em projeção dos rins (fisiológica).

A infecção pelo Mycobacterium tuberculosis determina várias reações inflamatórias, mediadas por células, no pulmão infectado: reações exsudativa (mediada por neutrófilos polimorfonucleares e macrófagos alveolares), produtiva (mediada por macrófagos alveolares e linfócitos com formação do granuloma), produtiva caseosa e cicatrizante $e^{(22)}$.

Nas doenças infecciosas, é possível que parte do FDG administrado seja absorvida pelo microorganismo causa- 


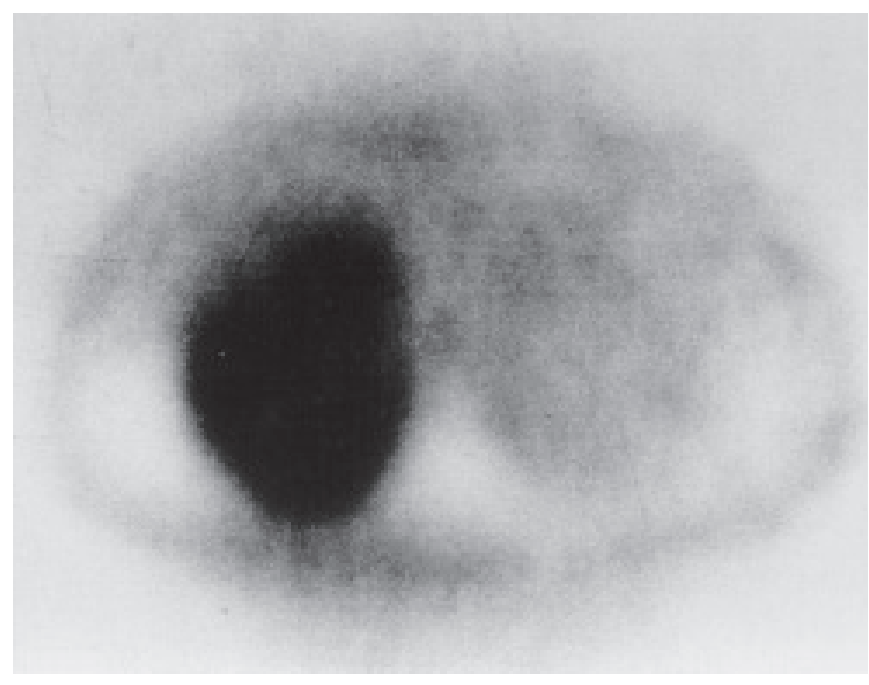

Figura $4 \boldsymbol{A}$ - PET: no corte transverso da PET, observa-se captação do 18F-FDG em pulmão direito (grau 2).

dor, desde que este se utilize de glicose como fonte de energia(8).

O Mycobacterium tuberculosis utiliza-se dos compostos do carbono para o seu crescimento em laboratório; assim, atuando como patógeno em diversos tecidos, assimila os metabólitos do hospedeiro para seu crescimento e multiplicação; é ainda capaz de assimilar, não só carboidratos, mas também lipídios e proteínas para a formação de lipases e proteases utilizadas em seu metabolismo(23).

A principal fonte de energia no metabolismo do $\mathrm{Myco-}$ bacterium tuberculosis é a glicose; esta, além de fornecer metabólitos intermediários para a biossíntese de macromoléculas, culmina na formação da enzima acetil-CoA e oxalato, do qual deriva o ácido cítrico necessário para o início do ciclo de Krebs e a produção de ATP.

Quando os resultados da PET foram comparados com os achados da TC (Tabela 2), observou-se que houve concordância em 153 dos 240 campos analisados (Figuras 4A e 4B). Houve discordância entre a PET e a TC em 87 campos. Nesses, os sinais sugestivos de atividade estiveram presentes na TC em 55 campos, que apresentaram captação negativa do FDG. Em 32 campos, houve captação do FDG na ausência dos sinais de atividade na TC, sugerindo que o processo metabólico estaria precedendo as alterações anatômicas nessas localizações.

A captação do FDG após o término do tratamento pode ser representativa do processo inflamatório que persiste após a eliminação do agente infeccioso ou presença de bacilos latentes. A explicação para esse comportamento talvez possa ocorrer em futuro próximo com o acompanhamento desses pacientes, para observação da evolução e possíveis recidivas. Dessa forma, poderemos avaliar a utilidade da PET nos pacientes com dificuldades para o diagnóstico da TB ou reativação da doença.

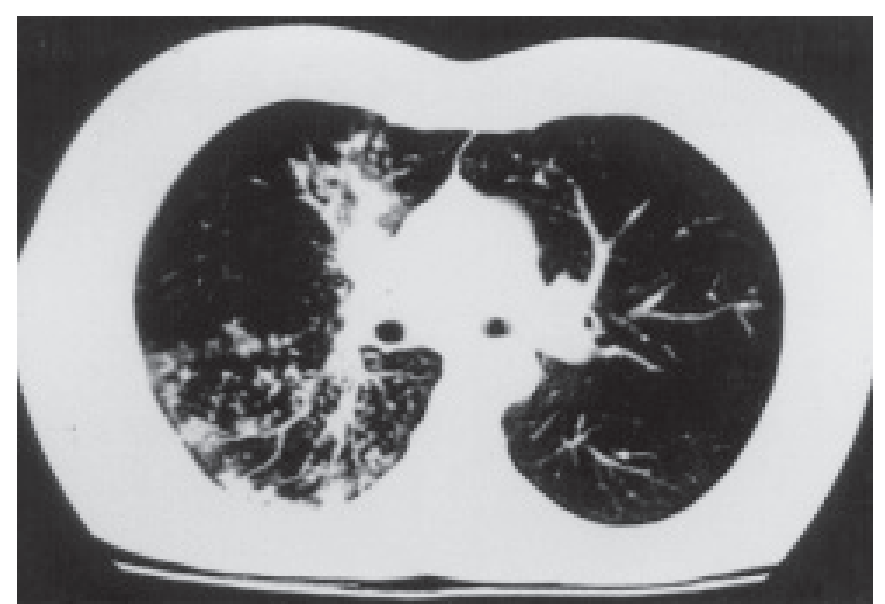

Figura 4B - TC de tórax do mesmo paciente: nódulos centrolobulares de distribuição segmentar, por vezes confluentes e aspecto de "arvore em florescência" em pulmão direito (extensão de grau 2 em porção posterior e de grau 1 em porção anterior).

A possibilidade de métodos que associem imagens obtidas por métodos de medicina nuclear com imagens da tomografia computadorizada ou ressonância magnética combinam informações fisiológicas e anatômicas das estruturas analisadas.

O presente estudo analisou os dois métodos separadamente. Todos os pacientes apresentaram captação positiva do FDG no início do tratamento, demonstrando que o metabolismo da glicose está aumentado na tuberculose ativa.

Embora tenha sido observada concordância entre os dois métodos, em alguns pacientes, o metabolismo aumentado da glicose foi observado pela PET em regiões nas quais não foram evidenciadas alterações estruturais na TC. Ainda, a TC pôde demonstrar, em determinados campos, alterações não observadas pela PET. Assim, os resultados da PET podem somar-se aos da TC na avaliação da tuberculose pulmonar e vice-versa.

\section{CONCLUSÕES}

Concluiu-se, com o presente estudo, que há captação do 18F-FDG através da PET em pacientes com tuberculose pulmonar ativa e que há concordância com os achados da tomografia computadorizada do tórax.

\section{AgradeCIMENTOS}

Ao Dr. José Cláudio Meneguetti, diretor do Serviço de Medicina Nuclear do InCor-HCFMUSP pela viabilização deste estudo e à Dra. Cláudia Maria Figueiredo, médica assistente do Serviço de Radiologia do HCFMUSP, pela colaboração na avaliação das imagens tomográficas. 


\section{REFERÊNCIAS}

1. World Health Organization, Global Tuberculosis Control WHO Report 2001. Geneva, Switzerland, WHO/CDS/TB/2001.287.

2. Brasil. Ministério da Saúde. Plano Nacional de Controle da Tuberculose. Manual de normas para o controle da tuberculose. Brasília, 2000.

3. Bombarda S, Figueiredo CM, Seiscento M, Soares-Júnior J, Funari MBG, Terra-Filho M. Imagem em tuberculose. J Pneumol 2001;27: 329-40.

4. Rigo P, Paulus P, Kaschten BJ, Hustinx R, Bury T, Jerusalem G, et al. Oncological applications of positron emission tomography with fluorine-18 fluorodeoxyglucose. Eur J Nucl Med 1996;23:1641-74.

5. Bury T, Dowlati A, Paulus P, Hustinx R, Radermecker M, Rigo P. Staging of non-small-cell lung cancer by whole-body fluorine-18 deoxyglucose positron emission tomography. Eur J Nucl Med 1996;23:204-6.

6. Marom EM, McAdams HP, Erasmus JJ, Goodman PC. Staging nonsmall cell lung cancer with whole-body PET. Radiology 1999;212: 803-9.

7. Lewis P, Griffin S, Marsden P, Gee T, Nunan T, Malsey M, et al. Wholebody 18F-fluorodeoxyglucose positron emission tomography in preoperative evaluation of lung cancer. Lancet 1994;344:1265-6.

8. Ichiya Y, Kuwabara Y, Otsuka M, Tahara T, Yoshikai T, Fukumura T, et al. Assessment of response to cancer therapy using fluorine-18-fluorodeoxyglucose and positron emission tomography. J Nucl Med 1991; 32:1655-60.

9. Flanagan FL, Dehdashti F, Siegel BA. PET in breast cancer. Semin Nucl Med 1998;28:290-302.

10. Al-Sugair A, Coleman RE. Applications of PET in lung cancer. Semin Nucl Med 1998;28:303-19.

11. Coleman RE. PET in lung cancer. J Nucl Med 1999;40:814-20.

12. Midthun DE, Jett JR. Lung tumors. In: Albert R, Spiro S, Jett J, editors. Comprehensive respiratory medicine. Denver: Mosby, 1999;43.124.
13. Leung AN. Pulmonary tuberculosis: the essentials. Radiology 1999; 210:307-22.

14. Lee KS, Hwang JW, Chung MP, Kim H, Kwon OJ. Utility of CT in the evaluation of pulmonary tuberculosis in patients without AIDS. Chest 1996;110:977.

15. Im JG, Itoh H, Shim YS, Lee JH, Ahn JA, Han MC, et al. Pulmonary tuberculosis: CT findings - Early active disease and sequential change with antituberculous therapy. Radiology 1993;186:653-60.

16. McAdams HP, Erasmus J, Winter JA. Radiologic manifestations of pulmonary tuberculosis. Radiol Clin North Am 1995;33:655-78.

17. Lee KS, Song KS, Lim PN, Kim PN, Lee BH. Adult-onset pulmonary tuberculosis: findings on chest radiographs and CT scans. AJR Am J Roentgenol 1993;160:753-8.

18. Pereira MG. Aferição dos eventos. In: Epidemiologia: teoria e prática. Rio de Janeiro: Guanabara Koogan, 1995;363-7.

19. Wagner HN. A brief history of positron emission tomography (PET). Semin Nucl Med 1998;28:213-20

20. Terra-Filho M, Bombarda S, Soares-Júnior J, Meneghetti JC. Tomografia por emissão de pósitrons (PET) no tórax: resultados preliminares de uma experiência brasileira. J Pneumol 2000;26:183-8.

21. Jones HA, Clark RJ, Rhodes CQ, Schofield JB, Krausz T, Haslett C. In vivo measurement of neutrophil activity in experimental lung inflammation. Am J Respir Crit Care Med 1994;149:1635-9.

22. Capellozi VL. Tuberculose. In: Brasileiro-Filho G, Bogliolo, editors. Patologia. Rio de Janeiro: Guanabara Koogan, 2000;320-2.

23. Ketai L, Hartshorne M. Potential uses of computed tomography-SPECT and computed tomography-coincidence fusion images of the chest. Clin Nucl Med 2001;26:433-41. 